\title{
Research on Fault Detection and Isolation Algorithm for Hexacopters Based on Detection Filter
}

\author{
Zhang Yumin ${ }^{1, a}$, Zhang Hongdi ${ }^{2, b}$ and Deng Junwu ${ }^{2, c}$ \\ ${ }^{1}$ School of Instrumentation Science and Opto-electronics Engineering, Beihang University, Beijing \\ 100191, China; \\ ${ }^{2}$ School of Instrumentation Science and Opto-electronics Engineering, Beihang University, Beijing \\ 100191, China. \\ a zhyminus @163.com, b hongdi442080@163.com, c929950063@qq.com
}

Keywords: Hexacopter, fault detection, detection filter, MATLAT simulation.

\begin{abstract}
Rotor UAV is a small, lightweight and low-cost unmanned vehicles. In recent years ,it has been widely used in production and daily life. With the rotor unmanned aerial vehicles more and more appear in people's work and life, UAV flight safety issues have become a concern. Many researches on controllability and fault-tolerant control strategy of rotor UAV are being carried out. This paper mainly designs a fault detection strategy based on the detection filter of the hexacopter. Firstly, establishing a mathematical model based on the kinematics and dynamics principles of hexacopter. Secondly, making an introduction for the basic principles of the detection filter. On the basis of the principles, a detection filter is designed to detect the faults which may occur on the actuators of the hexacopter. Finally, the performance of the fault detection method based on the detection filter is tested by simulation experiments
\end{abstract}

\section{Introduction}

In recent years, with the development of science and technology the application of small rotor unmanned aerial vehicle has begun to rise. Because of its small size, low cost and simple model, the rotor UAV has got a good development. And it has been playing an important role in the fields of power inspection, agricultural plant protection, aerial photography, game development, scientific research and so on. The security problem of rotor UAV has become a hot issue of people's attention. Many scholars have carried on the modeling and the control algorithm[1,2,3,4], the controllability $[5,6]$ and the fault-tolerant control[7,8,9] research for the rotor unmanned aerial vehicle

\section{Basic Principle and Mathematical Modeling of Hexacopter}

\subsection{The Basic Principles of Hexacopter}

The rotation of the six blades of the hexacopter aircraft provides lift and torque to control the position and attitude of the aircraft. The basic model of a hexacopter is shown below.

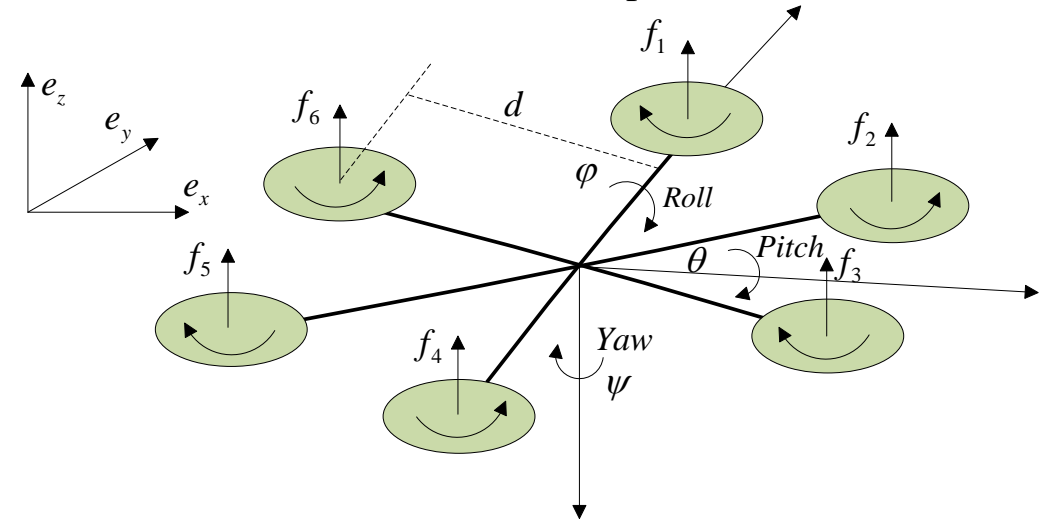

Fig. 1 The basic model of hexacopter 


\subsection{Establishment of Basic Coordinate System and Parameter Setting}

The ground coordinate system established in this paper is the northeast sky coordinate system. The basic parameters of the six rotorcraft are as follows:

Table 1 Basic parameter setting for hexacopter

\begin{tabular}{lccc}
\hline & Attitude Angle & Attitude Angular Velocity & Torque \\
\hline Around the X axis & $\varphi$ & $p$ & $L$ \\
Around the Y axis & $\theta$ & $q$ & $M$ \\
Around the Z axis & $\psi$ & $r$ & $N$ \\
\hline
\end{tabular}

According to the basic principle of the hexacopter, we can see that the lift and torque of a single rotor are proportional to the square of the speed of its motor. The lift coefficient is $b$ and the torque coefficient is $d$. Lift and torque are calculated as follows:

$$
\begin{aligned}
f_{i} & =b \omega_{i}^{2}(i=1,2,3,4,5,6) \\
\tau_{i} & =d \omega_{i}^{2}(i=1,2,3,4,5,6)
\end{aligned}
$$

The total lift of the hexacopter is provided by the six rotors, so the total lift can be expressed as follows:

$$
T_{b}=\sum_{i=0}^{6} f_{i}
$$

The torque expressions for the three axes of the hexacopter are as follows:

$$
\left[\begin{array}{l}
L \\
M \\
N
\end{array}\right]=\left[\begin{array}{l}
b \cdot l \cdot \sin \left(\frac{\pi}{3}\right) \cdot\left(\omega_{5}^{2}+\omega_{6}^{2}-\omega_{2}^{2}-\omega_{3}^{2}\right) \\
b \cdot l \cdot\left[\left(\omega_{1}^{2}-\omega_{4}^{2}\right)+\sin \left(\frac{\pi}{6}\right) \cdot\left(\omega_{2}^{2}+\omega_{6}^{2}-\omega_{3}^{2}-\omega_{5}^{2}\right)\right. \\
d \cdot\left(\omega_{1}^{2}-\omega_{2}^{2}+\omega_{3}^{2}-\omega_{4}^{2}+\omega_{5}^{2}-\omega_{6}^{2}\right)
\end{array}\right]
$$

The transfer matrix is:

$$
\operatorname{Rot}(\psi, \theta, \varphi)=\left[\begin{array}{ccc}
C \psi C \theta & C \psi S \theta S \varphi-S \psi S \varphi & C \psi S \theta S \varphi+S \psi S \varphi \\
S \psi C \theta & S \psi S \theta S \varphi+C \psi C \varphi & S \psi S \theta C \varphi-C \psi S \varphi \\
-S \theta & C \theta S \varphi & C \theta C \varphi
\end{array}\right]
$$

with $C=\cos , S=\sin$.

\subsection{Mathematical Modeling}

Based on the introduction of the above basic principles and formula derivation, it is possible to build a position model and attitude model for the hexacopter. According to Newton's first law, the displacement dynamic equation of the hexacopter in the ground coordinate is :

$$
m \cdot \overrightarrow{a_{e}}=\overrightarrow{G_{e}}+\overrightarrow{T_{e}}+\overrightarrow{R_{e}}
$$

According to Newton's second law, the rigid body's rotational dynamic equation is:

$$
I \vec{\varpi}=-\vec{\varpi} \times(I \vec{\varpi})+\vec{\Gamma}_{\text {tot }}
$$

with $I$ is moment of inertia, $\varpi$ is angular velocity, $\vec{\Gamma}_{\text {tot }}$ is the sum of moments.

For simpilicity, coupling and air resistance ban be ignore. And the simplified mathematical model and attitude model of the hexacopter are as follows:

$$
\left[\begin{array}{c}
\ddot{x} \\
\ddot{y} \\
\ddot{z}
\end{array}\right]=\left[\begin{array}{c}
-(\cos \varphi \sin \theta \cos \psi+\sin \varphi \sin \psi) \frac{\vec{T}_{b}}{m} \\
-(\cos \varphi \sin \theta \sin \psi-\sin \varphi \cos \psi) \frac{\vec{T}_{b}}{m} \\
-\cos \varphi \cos \theta \frac{\vec{T}_{b}}{m}+g
\end{array}\right],\left[\begin{array}{c}
\ddot{\varphi} \\
\ddot{\theta} \\
\ddot{\psi}
\end{array}\right]=\left[\begin{array}{c}
\frac{1}{I_{x}} \cdot b \cdot l \cdot \sin \left(\frac{\pi}{3}\right) \cdot\left(\omega_{5}{ }^{2}+\omega_{6}{ }^{2}-\omega_{2}{ }^{2}-\omega_{3}{ }^{2}\right) \\
\frac{1}{I_{y}} \cdot b \cdot l\left[\left(\omega_{1}{ }^{2}-\omega_{4}{ }^{2}\right)+\sin \left(\frac{\pi}{6}\right) \cdot\left(\omega_{2}{ }^{2}-\omega_{3}{ }^{2}+\omega_{6}{ }^{2}-\omega_{5}{ }^{2}\right)\right] \\
\frac{1}{I_{z}} \cdot d \cdot\left(\omega_{1}{ }^{2}-\omega_{2}{ }^{2}+\omega_{3}{ }^{2}-\omega_{4}{ }^{2}+\omega_{5}{ }^{2}-\omega_{6}{ }^{2}\right)
\end{array}\right]
$$




\section{Design of Fault Detection Algorithm Based on Detection Filter}

\subsection{Introduction for the Detection Filter}

This paper presents a method based on detection filter to detect the faults on the actuator of hexacopter. The use of detection filters for fault diagnosis is proposed by the American scholar Beard. The detection filter is a special observer operating in closed-loop mode, which fixes the output of the component and the actuator fault in a specific direction while limiting the fault output of the sensor to a plane. According to the specific design of the filter gain, you can complete the sensor and actuator fault diagnosis. The basic structure of the detection filter is shown in Fig.

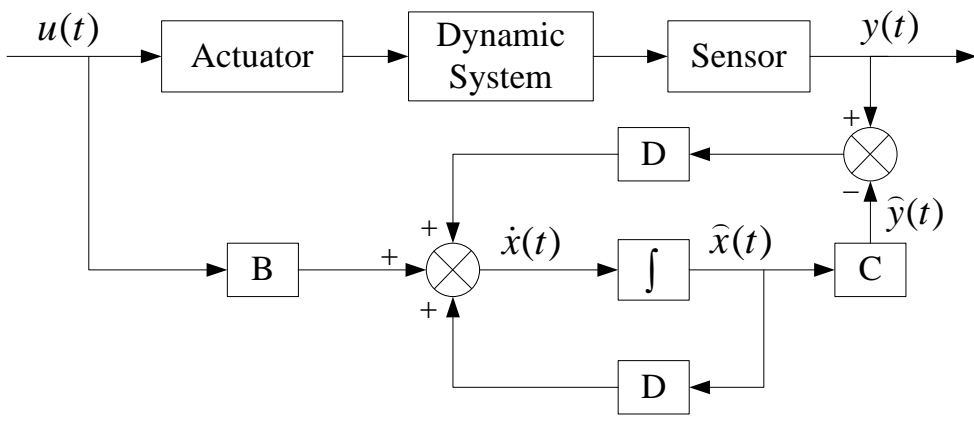

Fig. 2 Basic schematic of detection filter

The detection filter comprises a system dynamic model under normal operating conditions. The input to the model is the same as the input to the real system. The difference signal between the system sensor output and the model output is fed back to the model input via the gain matrix $\mathrm{D}[10]$.

\subsection{Design of the Detection Filter}

According to the basic principle of the detection filter, the fault can be detected when the filter gain matrix D satisfies the following two conditions.(1) $C e_{s}(t)$ maintains a fixed orientation in the output space. (2) All the eigenvalues of $(A-D C)$ can be arbitrarily configured. The eigenvalues and eigenvectors of $(A-D C)$ are determined by the following equation.

$$
\left(\lambda_{i} I-(A-D C)\right) v_{i}=0
$$

It is known from the observability of the system that $C v_{i}$ is independent of each other and covers the error variable state space. Therefore, the design of the fault vector can be written as a linear combination of closed-loop eigenvectors

$$
b_{i}=\sum_{i=1}^{n_{i}} a_{i} v_{i}
$$

The necessary and sufficient conditions for satisfying the condition of fault detectability (2) is that $\mathrm{C}$ and $\mathrm{B}$ are collinear while $i=1,2, \cdots, n_{i}$. Therefore, we can get $C v_{i}=C b_{i}$ by the standardization of $v_{i}$. The above theorem can constrain the closed-loop eigenvectors to ensure the unidirectionality of output errors. And then the fault vector $b_{i}$ can be configured as a feature vector of $(A-D C)$.

$$
\begin{aligned}
& (A-D C) f_{i}=\lambda_{i} f_{i} \\
& D C F=\left[\begin{array}{llll}
A_{1} b_{1}-\lambda_{1} b_{1} & A_{2} b_{2}-\lambda_{2} b_{2} & \cdots & A_{r} b_{r}-\lambda_{r} b_{r}
\end{array}\right] \\
& D=\left[\begin{array}{llll}
A_{1} b_{1}-\lambda_{1} b_{1} & A_{2} b_{2}-\lambda_{2} b_{2} & \cdots & A_{r} b_{r}-\lambda_{r} b_{r}
\end{array}\right](C F)^{+}+E\left(I-(C F)(C F)^{+}\right)
\end{aligned}
$$

Through the design of the filter, we can get the appropriate feedback gain matrix $\mathrm{D}$ so that the actuator fault can be detected and the output error is fixed in the direction of the fault vector.

\subsection{Design of fault diagnosis strategy based on detection filter for hexacopter}

This paper mainly aims at the fault detection design for actuator malfunction. When one of the hexacopter blades fails, the fault detection algorithm can detect the location of the fault in time. The state equation of six hexacopter is defined as:

$$
\begin{aligned}
& \dot{x}(t)=A x(t)+B u(t) \\
& y(t)=C x(t)
\end{aligned}
$$

with $u(t)$ is the square of the motor speed. 
The fault vector is $b_{i}=B \cdot e_{6 i}$. According to the feedback gain configuration method, the fault vector is configured as the eigenvector of $(A-D C)$. All the eigenvalues are taken as -1 , and a suitable $\mathrm{D}$ matrix can be calculated.

Fault vectors have been configured as the eigenvectors of $(A-D C)$ during the design of the filter gain matrices so that the fault can be separated by calculating the correlation of the residuals to the fault vector. According to the projection principle, the relationship between the two vectors can be expressed by the cosine of the angle between the two vectors.

$$
\cos \theta_{i}=\frac{\left|r^{T} b_{i}\right|}{\|r\|_{2}\left\|b_{i}\right\|_{2}}
$$

When the angle cosine of the $\mathrm{i}$-th fault vector is maximum, it can be determined that the $\mathrm{i}$-th actuator is most likely to fail.

\section{Simulation Experiment}

An experiment for the fault detection algorithm discussed above has been done. In order to simulate the fault state, a fault injection module was designed. The MATLAB simulation of the detection filter is shown in Fig. 3

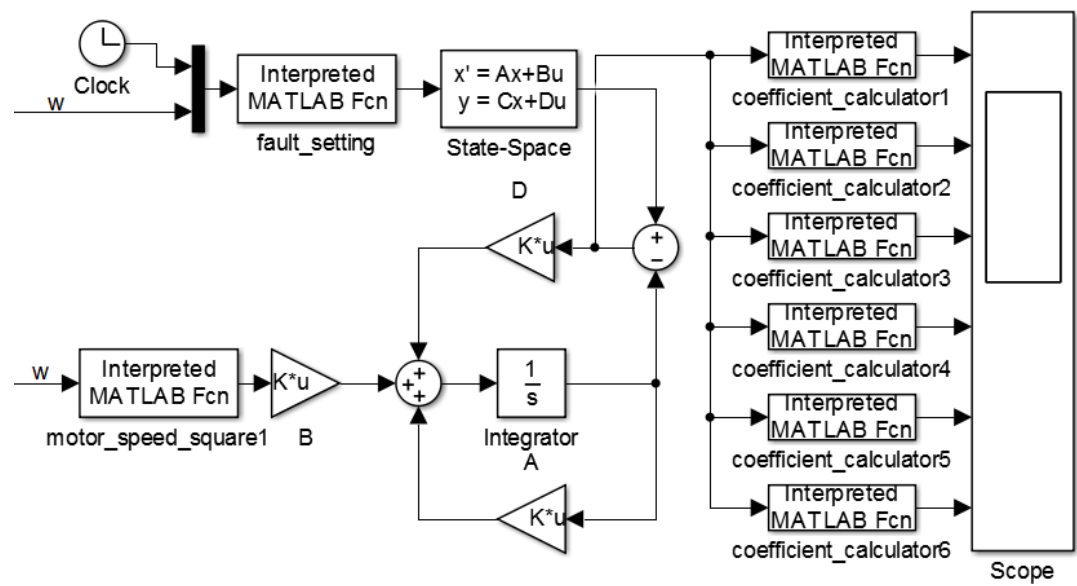

Fig. 3 Simulation of the detection filter

When the No.1 rotor fails in 5s, the detection result is shown in the Fig. 4. It can be seen from the figure that the residual vector and the fault vector corresponding to No. 1 motor and No. 4 motor is relatively strong. By magnifying the graph of the detection result, the Fig. 5 can be obtained.

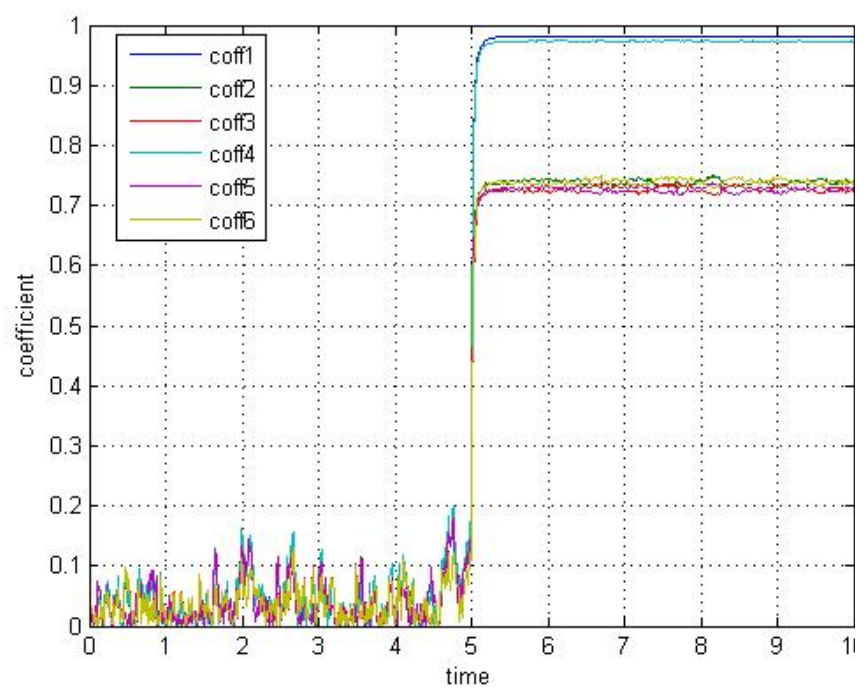

Fig. 4 Test results when motor 1 fails

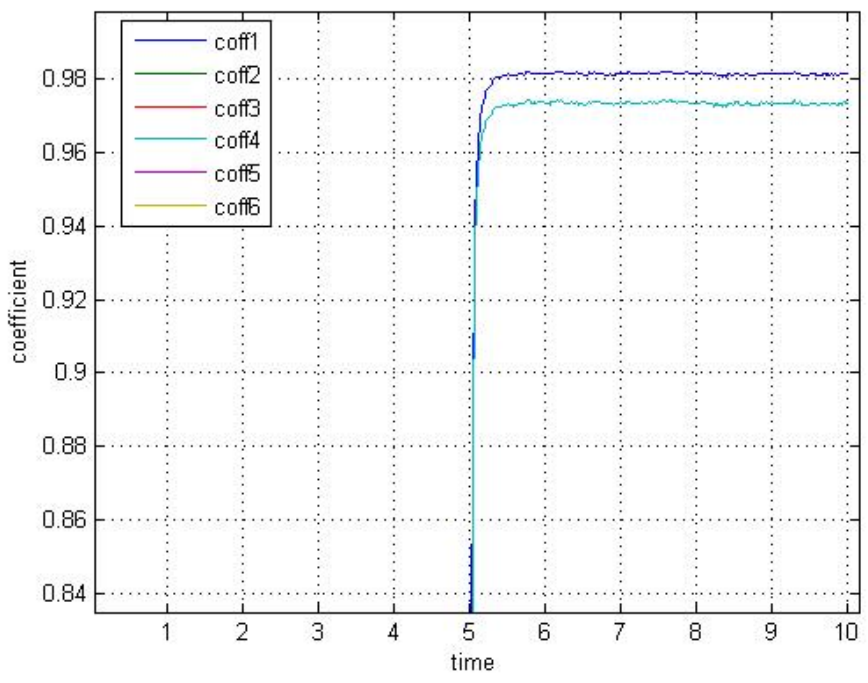

Fig. 5 Local amplification of the test results 
It can be seen from the figure that the residual vector has the strongest correlation with the fault vector corresponding to the No. 1 motor. So we can determine that the No. 1 rotor of the hexacopter is to fail. Test results are very accurate and the corresponding speed is within 0.5 seconds.

\section{Summary}

It can be seen from the test results that the fault detection method based on the detection filter has high detection speed and low false positive rate. Hexacopter in the flight process requires rapid feedback in order to adjust the flight attitude. The fault detection algorithm based on the detection filter has the advantages of simple calculation and fast response compared with other algorithms, so it is very suitable for hexacopter.

\section{Acknowledgements}

The author thanks the financial support of the National Natural Science Foundation of China, grant 61374131.

\section{References}

[1] Alaimo A, Artale V, Milazzo C, et al. Mathematical modeling and control of a hexacopter[C]//Unmanned Aircraft Systems (ICUAS), 2013 International Conference on. IEEE, 2013: 1043-1050.

[2] Artale V, Barbaraci G, Milazzo C, et al. Dynamic analysis of a hexacopter controlled via LQR-PI[C]//AIP Conference Proceedings. 2013.

[3] Alaimo A, Artale V, Milazzo C L R, et al. Pid controller applied to hexacopter flight[J]. Journal of Intelligent \& Robotic Systems, 2014, 73(1-4): 261-270.

[4] Bayrakceken M K, Yalcin M K, Arisoy A, et al. HIL simulation setup for attitude control of a quadrotor[C]//Mechatronics (ICM), 2011 IEEE International Conference on. IEEE, 2011: 354-357.

[5] Du G X, Quan Q, Cai K Y. Controllability Analysis and Degraded Control for a Class of Hexacopters Subject to Rotor Failures[J]. Journal of Intelligent \& Robotic Systems, 2013, 78(1):143-157.

[6] Mueller M W, D'Andrea R. Stability and control of a quadrocopter despite the complete loss of one, two, or three propellers[C]// IEEE International Conference on Robotics \& Automation. 2014:45-52.

[7] Schneider T, Ducard G, Rudin K, et al. Fault-tolerant Control Allocation for Multirotor Helicopters Using Parametric Programming[C]// International Micro Air Vehicle Conference and Flight Competition. 2012.

[8] Schneider T, Ducard G, Rudin K, et al. Fault-tolerant Control Allocation for Multirotor Helicopters Using Parametric Programming[C]// International Micro Air Vehicle Conference and Flight Competition. 2012.

[9] M. Saied, B. Lussier, I. Fantoni, et al. Fault tolerant control for multiple successive failures in an octorotor: Architecture and experiments[C]// Ieee/rsj International Conference on Intelligent Robots and Systems. IEEE, 2015.

[10] Zhou Donghua, Sun Youxian. Fault detection and diagnosis technology of control system [M]. Tsinghua University Press, 1994 